\title{
Boundary Continuity of Nonparametric Prescribed Mean Curvature Surfaces
}

\author{
Mozhgan Nora Entekhabi and Kirk E. Lancaster*
}

\begin{abstract}
We investigate the boundary behavior of variational solutions of Dirichlet problems for prescribed mean curvature equations at smooth boundary points where certain boundary curvature conditions are satisfied (which preclude the existence of local barrier functions). We prove that if the Dirichlet boundary data $\phi$ is continuous at such a point (and possibly nowhere else), then the solution of the variational problem is continuous at this point.
\end{abstract}

\section{Introduction}

Let $\Omega$ be a locally Lipschitz domain in $\mathbb{R}^{2}$ and define $N f=\nabla \cdot T f=\operatorname{div}(T f)$, where $f \in C^{2}(\Omega)$ and $T f=\nabla f / \sqrt{1+|\nabla f|^{2}}$. Let $H \in C^{1}(\bar{\Omega})$ satisfy the condition

$$
\left|\int_{\Omega} H \eta d x\right| \leq \frac{1}{2} \int_{\Omega}|D \eta| d x \quad \text { for all } \eta \in C_{0}^{1}(\Omega)
$$

(e.g., [10, (16.60)]). Here and throughout the paper, we adopt the sign convention that the curvature of $\Omega$ is nonnegative when $\Omega$ is convex. Consider the Dirichlet problem

$$
\begin{array}{cc}
N f=2 H & \text { in } \Omega, \\
f=\phi & \text { on } \partial \Omega .
\end{array}
$$

We wish to understand the boundary behavior of a solution of $11.2-1.3$.

If $\partial \Omega$ is smooth and the curvature of $\partial \Omega$ is greater than or equal to $2|H|$ at each point of $\partial \Omega$, then this problem is well-posed. If $\phi \in C^{0}(\partial \Omega)$, then there exists a unique solution of $(1.2-1.3)$ in $C^{2}(\Omega) \cap C^{0}(\bar{\Omega})$ and $f=\phi$ on $\partial \Omega$ (see 10 , Theorem 16.10]). On the other hand, one can choose a distinguished point $\mathcal{O} \in \partial \Omega$ and use the "gliding hump" construction as in [15] and [16, Theorem 3], in conjunction with [7, Theorem 2], to prove that there exist $\phi \in L^{\infty}(\partial \Omega)$ such that the (unique) variational solution $f$ of $(1.2)-(1.3)$ is in $C^{2}(\Omega)$ but $f$ is discontinuous at $\mathcal{O}$ and none of the radial limits of $f$ at $\mathcal{O}$ exist.

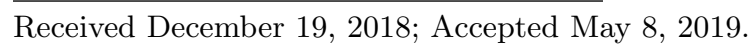

Communicated by Eiji Yanagida.

2010 Mathematics Subject Classification. Primary: 35J67; Secondary: 35J93, 53A10.

Key words and phrases. prescribed mean curvature, Dirichlet problem, boundary continuity.

*Corresponding author. 
When the appropriate geometric conditions (e.g., convexity of the domain in the case of the minimal surface equation on $\mathbb{R}^{2}$ ) are not satisfied, then the Dirichlet problem is illposed and a classical solution of $1.2-1.3$ may not exist (see, for example, 12 and 21. $\S 406])$. Interest in determining sufficient conditions for the existence of classical solutions of (1.2)-1.3 is long standing and one method is to impose "smallness" conditions on the Dirichlet boundary data $\phi$. When $H \equiv 0$ in 1.2 , an early result is A. Korn's classic 1909 paper [14]; J. C. C. Nitsche discusses some of the history of this problem for the minimal surface equation in [21, §285 \& §412]. G. Williams [25, 26], C. P. Lau [17], K. Hayasida and M. Nakatani [11], M. Bergner [1], J. Ripoll and F. Tomi [22 and many others have investigated limiting $\phi$ in order to prove a classical solution exists.

Rather than imposing a "smallness" condition on the Dirichlet data $\phi$ and trying to obtain a classical solution of $1.2-(1.3)$, we wish to impose a (local) condition on the curvature of the domain, place no restrictions on the Dirichlet data $\phi \in L^{\infty}(\partial \Omega)$ and prove that the variational solution $f$ extends to be continuous at a point $\mathcal{O}$ of $\partial \Omega$ (or on an open subset $\Gamma$ of $\partial \Omega$ ) in the sense that $f \in C^{0}\left(\Omega \cup\{\mathcal{O}\}\right.$ ) (or $f \in C^{0}(\Omega \cup \Gamma)$ ) when $\phi$ is continuous at $\mathcal{O}$ (or on $\Gamma$ ). In general, no classical solution may exist and the variational solution is the best approximation to a classical solution.

We shall assume that $\partial \Omega$ is smooth, $\mathcal{O} \in \partial \Omega$ is a distinguished point, the curvature $\Lambda$ of $\partial \Omega$ satisfies

$$
\Lambda(\mathbf{x})<-2|H(\mathbf{x})| \quad \text { for } \mathbf{x} \in \partial \Omega,|\mathbf{x}-\mathcal{O}|<\delta
$$

for some $\delta>0$ and $\phi \in L^{\infty}(\partial \Omega)$. In Theorem 1.1, we prove that the radial limits $R f(\cdot)$ of $f$ exist at $\mathcal{O}$ if $\phi$ is discontinuous at $\mathcal{O}$ (even if $\phi$ does not have one-sided limits at $\mathcal{O}$ ). As a consequence of the existence of radial limits, we prove in Corollary 1.2 that the (unique) variational solution $f \in C^{2}(\Omega)$ of $\left.(1.2)-1.3\right)$ is continuous at $\mathcal{O}$ if $\phi$ is continuous at $\mathcal{O}$. In Corollary 1.3 , we assume $H \equiv 0$, relax (1.4) slightly and obtain the same result as in Corollary 1.2 .

The idea of the proof is to describe the graph of $f$ parametrically in isothermal coordinates, prove that it is uniformly continuous on its (open) parameter domain and therefore extends uniquely to a continuous function on the closure of the parameter domain. In the case of Corollaries 1.2 and 1.3 , this is equivalent to proving that $f$ is uniformly continuous on the intersection of $\Omega$ and an open neighborhood $U$ of $\mathcal{O}$ and therefore $f$ extends uniquely to a continuous function on $\overline{\Omega \cap U}$. Of course, just as in 2, Theorem 4.2], 20 and 24], this extension of $f$ needs not equal $\phi$. Differences between our results and those, for example, in the papers by Bourni, Lin and Simon are the additional requirements imposed on the domain or the boundary data; 2 requires the graph of $\phi$ to be a specific type of limit of the graphs of $C^{1, \alpha}$ functions, 20 requires $\phi$ to be Lipschitz and 24 requires $H \equiv 0, \partial \Omega$ to be $C^{4}$ and $\phi$ to be Lipschitz. In Example 1.4, we present an illustration 
of the value of symmetry and prove that $f$ can be continuous at $\mathcal{O}$ even if $\phi$ is not; this illustration uses the same domain as that mentioned in 24.

In the remaining case where $-2|H(\mathcal{O})| \leq \Lambda(\mathcal{O})<2|H(\mathcal{O})|$, the behavior of the variational solution at $\mathcal{O}$ is unknown. The Dirichlet problem $1.2-(1.3)$ does not have a classical solution in $C^{2}(\Omega) \cap C^{0}(\bar{\Omega})$ for all $\phi \in C^{\infty}(\partial \Omega)$ (see 10 , Corollary 14.13]) and the standard gliding hump argument does not work in this situation. On the other hand, not all of the comparison functions needed here are available and so the conclusions of Corollaries 1.2 and 1.3 may not hold. Moonies ( [9, 18], see also [19]) are not bounded below but do illustrate some properties of this case, with $H \equiv 1, \Lambda \equiv-2$ on one component of the boundary and $\Lambda \equiv 1 / R, 1 / 2<R<1$, on the other component. As in 13, these cases illustrate the strong differences between uniformly elliptic (genre zero) and prescribed mean curvature (genre two) equations (see also Remark 2.7); for Laplace's equation in $\mathbb{R}^{2}$, for example, boundary curvature would play no role in the solvability of Dirichlet problems and the gliding hump construction could always be used, exactly as in the first case above.

At a point $\mathbf{y} \in \partial \Omega$, we let $\alpha(\mathbf{y})$ and $\beta(\mathbf{y})$ be the angles which the tangent rays to $\partial \Omega$ at $\mathbf{y}$ make with the positive $x$-axis such that

$$
\{\mathbf{y}+r(\cos \theta, \sin \theta): 0<r<\epsilon(\theta), \alpha(\mathbf{y})<\theta<\beta(\mathbf{y})\} \subset \Omega \cap B_{\delta}(\mathbf{y})
$$

for some $\delta>0$ and some function $\epsilon(\cdot):(\alpha(\mathbf{y}), \beta(\mathbf{y})) \rightarrow(0, \delta)$. If $\partial \Omega$ is smooth at $\mathbf{y}$, $\beta(\mathbf{y})=\alpha(\mathbf{y})+\pi$. For $\theta \in(\alpha(\mathbf{y}), \beta(\mathbf{y})), R f(\theta, \mathbf{y})=\lim _{r \downarrow 0} f(\mathbf{y}+r(\cos \theta, \sin \theta))$ if this limit exists. Also $R f(\alpha(\mathbf{y}), \mathbf{y})=\lim _{\Gamma_{1}(\mathbf{y}) \ni \mathbf{x} \rightarrow \mathbf{y}} f(\mathbf{x})$ if this limit exists and $R f(\beta(\mathbf{y}), \mathbf{y})=$ $\lim _{\Gamma_{2}(\mathbf{y}) \ni \mathbf{x} \rightarrow \mathbf{y}} f(\mathbf{x})$ if this limit exists, where $\partial \Omega \cap B_{\delta}(\mathbf{y}) \backslash\{\mathbf{y}\}$ consists of disjoint, open $\operatorname{arcs} \Gamma_{1}(\mathbf{y})$ and $\Gamma_{2}(\mathbf{y})$ whose tangent rays approach the rays $\theta=\alpha(\mathbf{y})$ and $\theta=\beta(\mathbf{y})$ respectively, as the point $\mathbf{y}$ is approached. $\left(\Gamma_{1}(\mathbf{y})\right.$ (lower curve) and $\Gamma_{2}(\mathbf{y})$ (upper curve) are illustrated in Figure 1.1 in a case where $\beta(\mathbf{y})-\alpha(\mathbf{y})=\pi$.)

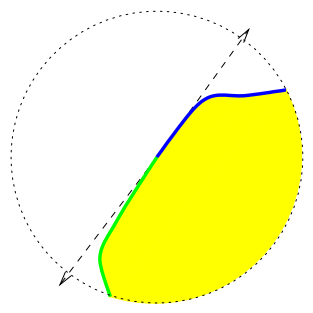

Figure 1.1: $\Gamma_{1}(\mathbf{y})$ (green); $\Gamma_{2}(\mathbf{y})$ (blue); $\Omega \cap B_{\delta}(\mathbf{y})$ (yellow).

Theorem 1.1. Suppose $\Omega$ is a locally Lipschitz domain in $\mathbb{R}^{2}, \Gamma$ is a $C^{2, \lambda}$ open subset of $\partial \Omega$ for some $\lambda \in(0,1)$. Suppose either (a) $H \equiv 0$ in a neighborhood of $\Gamma$, the curvature $\Lambda$ of $\Gamma$ is nonpositive and vanishes at only a finite number of points of $\Gamma$, at each of which (1.5) holds, or (b) the curvature $\Lambda(\mathbf{x})$ of $\Gamma$ at $\mathbf{x}$ is less than $-2|H(\mathbf{x})|$ for $\mathbf{x} \in \Gamma$. Let 
$f \in C^{2}(\Omega) \cap L^{\infty}(\Omega)$ satisfy $N f=2 H$ in $\Omega$. Suppose that $\mathbf{y} \in \Gamma$. Then the limits

$$
\lim _{\Gamma_{1}(\mathbf{y}) \ni \mathbf{x} \rightarrow \mathbf{y}} f(\mathbf{x})=z_{1} \quad \text { and } \quad \lim _{\Gamma_{2}(\mathbf{y}) \ni \mathbf{x} \rightarrow \mathbf{y}} f(\mathbf{x})=z_{2}
$$

exist, $R f(\theta, \mathbf{y})$ exists for each $\theta \in[\alpha(\mathbf{y}), \beta(\mathbf{y})], R f(\cdot, \mathbf{y}) \in C^{0}([\alpha(\mathbf{y}), \beta(\mathbf{y})])$, and $R f(\cdot, \mathbf{y})$ behaves in one of the following ways:

(i) $R f(\cdot, \mathbf{y})=z_{1}$ is a constant function and $f$ is continuous at $\mathbf{y}$.

(ii) There exist $\alpha_{1}$ and $\alpha_{2}$ so that $\alpha(\mathbf{y}) \leq \alpha_{1}<\alpha_{2} \leq \beta(\mathbf{y}), R f=z_{1}$ on $\left[\alpha(\mathbf{y}), \alpha_{1}\right]$, $R f=z_{2}$ on $\left[\alpha_{2}, \beta(\mathbf{y})\right]$ and $R f$ is strictly increasing (if $z_{1}<z_{2}$ ) or strictly decreasing (if $z_{1}>z_{2}$ ) on $\left[\alpha_{1}, \alpha_{2}\right]$.

Corollary 1.2. Suppose $\Omega$ is a locally Lipschitz domain in $\mathbb{R}^{2}, \Gamma$ is a $C^{2, \lambda}$ open subset of $\partial \Omega$ for some $\lambda \in(0,1)$, the curvature $\Lambda(\mathbf{x})$ of $\Gamma$ at $\mathbf{x}$ is less than $-2|H(\mathbf{x})|$ for $\mathbf{x} \in \Gamma$. Suppose $\phi \in L^{\infty}(\partial \Omega), \mathbf{y} \in \Gamma$, either $\phi$ is symmetric with respect to a line through $\mathbf{y}$ or $\phi$ is continuous at $\mathbf{y}$, and $f \in B V(\Omega)$ minimizes

$$
J(u)=\int_{\Omega} \sqrt{1+|D u|^{2}} d \mathbf{x}+\int_{\Omega} 2 H u d \mathbf{x}+\int_{\partial \Omega}|u-\phi| d s
$$

for $u \in B V(\Omega)$. Then $f \in C^{0}(\Omega \cup\{\mathbf{y}\})$. If $\phi \in C^{0}(\Gamma)$, then $f \in C^{0}(\Omega \cup \Gamma)$.

When $H \equiv 0$, the strict inequality 1.4 can be relaxed.

Corollary 1.3. Suppose $\Omega$ is a locally Lipschitz domain in $\mathbb{R}^{2}, \Gamma$ is a $C^{2, \lambda}$ open subset of $\partial \Omega$ for some $\lambda \in(0,1)$, and the curvature $\Lambda$ of $\Gamma$ is nonpositive and vanishes, at most, at a finite number of points of $\Gamma$. For each point $\mathbf{x}_{0} \in \Gamma$ at which $\Lambda\left(\mathbf{x}_{0}\right)=0$, suppose there exist $C>0$ and $\delta>0$ such that

$$
|\Lambda(\mathbf{x})| \geq C\left|\mathbf{x}-\mathbf{x}_{0}\right|^{\lambda} \quad \text { for } \mathbf{x} \in \Gamma,\left|\mathbf{x}-\mathbf{x}_{0}\right|<\delta .
$$

Suppose $\phi \in L^{\infty}(\partial \Omega), \mathbf{y} \in \Gamma$, either $\phi$ is symmetric with respect to a line through $\mathbf{y}$ or $\phi$ is continuous at $\mathbf{y}$, and $f \in B V(\Omega)$ minimizes

$$
J(u)=\int_{\Omega} \sqrt{1+|D u|^{2}} d \mathbf{x}+\int_{\partial \Omega}|u-\phi| d s
$$

for $u \in B V(\Omega)$. Then $f \in C^{0}(\Omega \cup\{\mathbf{y}\})$. If $\phi \in C^{0}(\Gamma)$, then $f \in C^{0}(\Omega \cup \Gamma)$.

Example 1.4. Let $\Omega=\left\{(x, y) \in \mathbb{R}^{2}: 1<(x+1)^{2}+y^{2}<\cosh ^{2}(1)\right\}$ and $\phi(x, y)=$ $\sin \left(\frac{\pi}{x^{2}+y^{2}}\right)$ for $(x, y) \neq(0,0)$ (see Figure 1.2 for a rough illustration of the graph of $\left.\phi\right)$. Set $\mathcal{O}=(0,0)$ and $H \equiv 0$. Let $f \in C^{2}(\Omega)$ minimize 1.6$)$ over $B V(\Omega)$. Then Corollary 1.2 (with $\mathbf{y}=\mathcal{O}$ ) implies $f \in C^{0}(\bar{\Omega})$, even though $\phi$ has no limit at $\mathcal{O}$. 

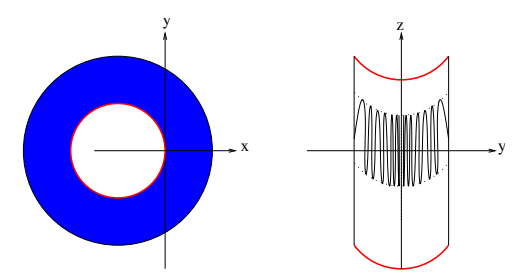

Figure 1.2: $\Omega$ and part of the graph of $\phi$.

\section{Proofs}

Let $Q$ be the operator on $C^{2}(\Omega)$ given by

$$
Q f(\mathbf{x}) \stackrel{\text { def }}{=} N f(\mathbf{x})-2 H(\mathbf{x}), \quad \mathbf{x} \in \Omega .
$$

Let $\nu$ be the exterior unit normal to $\partial \Omega$, defined almost everywhere on $\partial \Omega$. At every point $\mathbf{y} \in \partial \Omega$ for which $\partial \Omega$ is a $C^{1}$ curve in a neighborhood of $\mathbf{y}, \widehat{\nu}$ denotes a continuous extension of $\nu$ to a neighborhood of $\mathbf{y}$. We shall adopt the same convention as that adopted in [3, p. 178] regarding the meaning of the notation " $T \psi$ " and " $T \psi \cdot \nu$ " at points of $\partial \Omega$; thus, for example, the equation $T \psi(\mathbf{y}) \cdot \nu(\mathbf{y})= \pm 1$ at a point $\mathbf{y} \in \partial \Omega$ means

$$
\lim _{\Omega \ni \mathbf{x} \rightarrow \mathbf{y}} \frac{\nabla \psi(\mathbf{x}) \cdot \widehat{\nu}(\mathbf{x})}{\sqrt{1+|\nabla \psi(\mathbf{x})|^{2}}}= \pm 1
$$

and $T \psi(\mathbf{y})= \pm \nu(\mathbf{y})$ means $\lim _{\Omega \ni \mathbf{x} \rightarrow \mathbf{y}} T \psi(\mathbf{x})= \pm \nu(\mathbf{y})$.

Definition 2.1. Given a locally Lipschitz domain $\Omega$, an upper Bernstein pair $\left(U^{+}, \psi^{+}\right)$ for a curve $\Gamma \subset \partial \Omega$ and a function $H$ in 2.1) is a domain $U^{+}$and a function $\psi^{+} \in$ $C^{2}\left(U^{+}\right) \cap C^{0}\left(\overline{U^{+}}\right)$such that $\Gamma \subset \partial U^{+}, \nu$ is the exterior unit normal to $\partial U^{+}$at each point of $\Gamma$ (i.e., $U^{+}$and $\Omega$ lie on the same side of $\Gamma$ ), $Q \psi^{+} \leq 0$ in $U^{+}$, and $T \psi^{+} \cdot \nu=1$ almost everywhere on $\Gamma$ in the same sense as in [3]; that is, for almost every $\mathbf{y} \in \Gamma$,

$$
\lim _{U^{+} \ni \mathbf{x} \rightarrow \mathbf{y}} \frac{\nabla \psi^{+}(\mathbf{x}) \cdot \widehat{\nu}(\mathbf{x})}{\sqrt{1+\left|\nabla \psi^{+}(\mathbf{x})\right|^{2}}}=1 .
$$

Definition 2.2. Given a domain $\Omega$ as above, a lower Bernstein pair $\left(U^{-}, \psi^{-}\right)$for a curve $\Gamma \subset \partial \Omega$ and a function $H$ in (2.1) is a domain $U^{-}$and a function $\psi^{-} \in C^{2}\left(U^{-}\right) \cap$ $C^{0}\left(\overline{U^{-}}\right)$such that $\Gamma \subset \partial U^{-}, \nu$ is the exterior unit normal to $\partial U^{-}$at each point of $\Gamma$ (i.e., $U^{-}$and $\Omega$ lie on the same side of $\Gamma$ ), $Q \psi^{-} \geq 0$ in $U^{-}$, and $T \psi^{-} \cdot \nu=-1$ almost everywhere on $\Gamma$ (in the same sense as above).

The existence of Bernstein pairs is established in Lemmas 2.4 and 2.5 . 
Lemma 2.3. Let $a<b, \lambda \in(0,1), \psi \in C^{2, \lambda}([a, b])$ and $\Gamma=\left\{(x, \psi(x)) \in \mathbb{R}^{2}: x \in[a, b]\right\}$ such that $\psi^{\prime}(x)<0$ for $x \in[a, b], \psi^{\prime \prime}(x)<0$ for $x \in[a, b] \backslash J$, there exist $C_{1}>0$ and $\epsilon_{1}>0$ such that if $\bar{x} \in J$ and $|x-\bar{x}|<\epsilon_{1}$, then $\psi^{\prime \prime}(x) \leq-C_{1}|x-\bar{x}|^{\lambda}$, where $J$ is a finite subset of $(a, b)$. Then there exists an open set $U \subset \mathbb{R}^{2}$ with $\Gamma \subset \partial U$ and a function $h \in C^{2}(U) \cap C^{0}(\bar{U})$ such that $\partial U$ is a closed, $C^{2, \lambda}$ curve, $\Gamma$ lies below $U$ in $\mathbb{R}^{2}$ (i.e., the exterior unit normal $\nu=\left(\nu_{1}(x), \nu_{2}(x)\right)$ to $\partial U$ satisfies $\nu_{2}(x)<0$ for $\left.a \leq x \leq b\right), N h=0$ in $U$ and $T h \cdot \nu=1$ almost everywhere on an open subset of $\partial U^{+}$containing $\bar{\Gamma}$ (i.e., 2.2) holds).

Proof. We may assume that $a, b>0$. There exists $c>b$ and $k \in C^{2, \lambda}([-c, c])$ with $k(-x)=k(x)$ for $x \in[0, c]$ such that $k(x)=-\psi(x)$ for $x \in[a, b], k^{\prime \prime}(x)>0$ for $x \in$ $[-c, c] \backslash J$, where $J$ is a finite set, $k^{\prime \prime}(0)>0$, and the set

$$
K=\left\{(x, k(x)) \in \mathbb{R}^{2}: x \in[-c, c]\right\}
$$

is strictly concave (i.e., $t k\left(x_{1}\right)+(1-t) k\left(x_{2}\right)>k\left(t x_{1}+(1-t) x_{2}\right)$ for each $t \in(0,1)$ and $x_{1}, x_{2} \in[-c, c]$ with $\left.x_{1} \neq x_{2}\right)$. From [6, pp. 1063-1065], we can construct a domain $\Omega(K, l)$ such that $K \subset \partial \Omega(K, l)$ and $\Omega(K, l)$ lies below $K$ (i.e., the outward unit normal to $\Omega(K, l)$ at $(x, k(x))$ is $\nu(x)=\left(-k^{\prime}(x), 1\right) / \sqrt{1+\left(k^{\prime}(x)\right)^{2}}$; see 6 , Figure 4]) and a function $F^{+} \in C^{2}(\Omega(K, l)) \cap C^{0}(\overline{\Omega(K, l)})$ such that $N F^{+}=0$ and the function

$$
\mu(\mathbf{x}) \stackrel{\text { def }}{=} \frac{\left(\nabla F^{+}(\mathbf{x}),-1\right)}{\sqrt{1+\left|\nabla F^{+}(\mathbf{x})\right|^{2}}}, \quad \mathbf{x} \in \Omega(K, l),
$$

extends continuously to a function $\widehat{\mu}$ on $\Omega(K, l) \cup K$ and $\widehat{\mu}(x, k(x)) \cdot \nu(x)=1$ for $x \in[-c, c]$. Now let $V$ be an open subset of $\Omega$ with $C^{2, \lambda}$ boundary such that $\{(x,-\psi(x)): x \in$ $[a, b]\} \subset \partial V$ and $\partial V \cap(\partial \Omega(K, l) \backslash K)=\emptyset$ and then let $U=\{(x,-y):(x, y) \in V\}$ and $h(x, y)=F^{+}(x,-y)$ for $(x, y) \in \bar{U}$.

Lemma 2.4. Suppose $\Omega$ is a locally Lipschitz domain in $\mathbb{R}^{2}, \Gamma$ is a $C^{2, \lambda}$ open subset of $\partial \Omega$ for some $\lambda \in(0,1)$, and the curvature $\Lambda$ of $\Gamma$ is either negative or nonpositive, vanishes only at finite number of points of $\Gamma$ and 1.5 holds at each such point. Let $\mathbf{y} \in \Gamma$. Then there exist $\delta>0$ and upper and lower Bernstein pairs $\left(U^{ \pm}, \psi^{ \pm}\right)$for $\left(\Gamma \cap B_{\delta}(\mathbf{y}), 0\right)$.

Proof. Let $\Omega \subset \mathbb{R}^{2}$ be an open set, $\Gamma \subset \partial \Omega$ be a $C^{2, \lambda}$ curve and $\mathbf{y} \in \Gamma$ be a point at which we wish to have upper and lower Bernstein pairs for $H \equiv 0$. Notice that (1.5) implies that the "curvature" condition in Lemma 2.3 (i.e., after rotating $\Omega$, the condition that if $\Lambda(\bar{x}, \psi(\bar{x}))=0$, then $\psi^{\prime \prime}(x) \leq-C_{1}|x-\bar{x}|^{\lambda}$ when $x$ is near $\left.\bar{x}\right)$ is satisfied. Choose a neighborhood $V$ of $\mathbf{y}$ and a rigid motion $\zeta: \mathbb{R}^{2} \rightarrow \mathbb{R}^{2}$ such that $\Sigma \stackrel{\text { def }}{=} V \cap \partial \Omega \subset \Gamma$ and the curve $\zeta(\Sigma)$ satisfies the hypotheses of Lemma 2.3. Let $U$ and $h$ be as given in the conclusion of Lemma 2.3. Then $\left(\zeta^{-1}(U), h \circ \zeta\right)$ will be an upper Bernstein pair for $\Sigma$ and $H \equiv 0$ and $\left(\zeta^{-1}(U),-h \circ \zeta\right)$ will be a lower Bernstein pair for $\Sigma$ and $H \equiv 0$. 
Lemma 2.5. Suppose $\Omega$ is a $C^{2, \lambda}$ domain in $\mathbb{R}^{2}$ for some $\lambda \in(0,1), \mathbf{y} \in \partial \Omega$ and $\Lambda(\mathbf{y})<-2|H(\mathbf{y})|$, where $\Lambda(\mathbf{y})$ denotes the curvature of $\partial \Omega$ at $\mathbf{y}$. Then there exist $\delta>0$ and upper and lower Bernstein pairs $\left(U^{ \pm}, \psi^{ \pm}\right)$for $(\Gamma, H)$, where $\Gamma=B_{\delta}(\mathbf{y}) \cap \partial \Omega$.

Proof. We may assume $H \not \equiv 0$ in any neighborhood of $\mathbf{y}$ since Lemma 2.4 covers this case. There exists $\delta_{1}>0$ such that $\Lambda(\mathbf{x})<-2|H(\mathbf{x})|$ for each $\mathbf{x} \in \partial \Omega \cap B_{\delta_{1}}(\mathbf{y})$. There exists a $\delta_{2} \in\left(0, \delta_{1} / 2\right)$ such that

$$
\Lambda_{0} \stackrel{\text { def }}{=} \sup \left\{\Lambda(\mathbf{x}): \mathbf{x} \in \partial \Omega \cap B_{\delta_{2}}(\mathbf{y})\right\}<\inf \left\{-2|H(\mathbf{x})|: \mathbf{x} \in \bar{\Omega} \cap B_{\delta_{2}}(\mathbf{y})\right\} \stackrel{\text { def }}{=}-2 H_{0} .
$$

Fix $c_{0} \in\left(-1 /\left(4 H_{0}\right), 0\right)$ and set

$$
r_{1}=\frac{1-\sqrt{1+4 c_{0} H_{0}}}{2 H_{0}} \quad \text { and } \quad r_{2}=\frac{1+\sqrt{1+4 c_{0} H_{0}}}{2 H_{0}} .
$$

Let $\mathbf{q} \in \partial \Omega \cap B_{\delta_{2}}(\mathbf{y})$ and set $\mathbf{p}=\mathbf{p}(\mathbf{q})=\mathbf{q}+r_{1} \nu(\mathbf{q})$, define $\widehat{r}(\mathbf{x})=\widehat{r}_{\mathbf{q}}(\mathbf{x})=|\mathbf{x}-\mathbf{p}|$ and consider the annulus $\mathcal{A}=\mathcal{A}(\mathbf{q}) \stackrel{\text { def }}{=}\left\{\mathbf{x} \in \mathbb{R}^{2}: r_{1} \leq \widehat{r}(\mathbf{x}) \leq r_{2}\right\}$; let $\nu_{\mathbf{q}}$ denote the exterior unit normal to $\mathcal{A}(\mathbf{q})$. Let $z=h(\widehat{r})\left(=h_{\mathbf{q}}\left(\widehat{r}_{\mathbf{q}}\right)\right)$ be a unduloid surface (see for example $[9,16])$ defined on the annulus $\mathcal{A}(\mathbf{q})$ which has constant mean curvature $-H_{0}<0$ and becomes vertical at $\widehat{r}=r_{1}, r_{2}$ (with $T h_{\mathbf{q}} \cdot \nu_{\mathbf{q}}=1$ on $\widehat{r}_{\mathbf{q}}=r_{1}$ in the limiting sense (i.e., 2.2 holds)). Notice that $\mathcal{A}(\mathbf{q})$ touches $\partial \Omega$ at $\mathbf{q}$ and so there exists $0<\delta_{3}<\min \left\{\delta_{2} / 2, r_{2}-r_{1}\right\}$ such that $h^{\prime}(r) \leq 0$ if $r_{1}<r<r_{1}+\delta_{3}$ and for each $\mathbf{q} \in \partial \Omega \cap B_{\delta_{3}}(\mathbf{y}),\left\{\mathbf{x} \in B_{\delta_{3}}(\mathbf{y}):|\mathbf{x}-\mathbf{p}(\mathbf{q})|=r_{1}\right\} \subset \Omega \cup\{\mathbf{q}\}$ (see Figure 2.1). We shall assume that $\delta_{3}$ is picked small enough that the curvature of $\partial B_{\delta_{3}}(\mathbf{y})$ is larger than $\sup _{\Omega} 2|H|$.

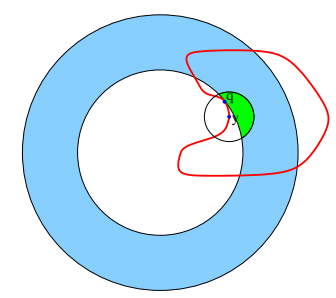

Figure 2.1: $\partial \Omega\left(\right.$ red); $\mathcal{A}(\mathbf{q})$ (blue); $U=\Omega \cap B_{\delta_{3}}(\mathbf{y})$ (green).

Set $z_{1}=h\left(r_{1}\right)-h\left(r_{2}\right)\left(=1 /\left(2 H_{0}\right)\right)$ and fix $z_{2}>z_{1}$. Let $\psi^{+}$be the variational solution of the Dirichlet problem $N \psi^{+}=-2 H_{0}$ in $U=\Omega \cap B_{\delta_{3}}(\mathbf{y}), \psi^{+}=z_{2}$ on $\Gamma=B_{\delta_{3}}(\mathbf{y}) \cap \partial \Omega$ and $\psi^{+}=0$ on $\partial U \backslash \Gamma$. Notice that $\psi^{+}$assumes its Dirichlet boundary value (of zero) on $\partial U \backslash \bar{\Gamma}$. For each $\mathbf{q} \in \Gamma$, we have

$$
\psi^{+}(\mathbf{x})=0<h_{\mathbf{q}}\left(\widehat{r}_{\mathbf{q}}(\mathbf{x})\right) \text { for all } \mathbf{x} \in \partial U \cap \mathcal{A}(\mathbf{q})
$$

and $T h_{\mathbf{q}} \cdot \nu_{\mathbf{q}}=1$ on $\widehat{r}_{\mathbf{q}}(\mathbf{x})=r_{1}$; it follows from 8, Theorem 5.1] that $\psi^{+}(\mathbf{x}) \leq z_{1}$ for every $\mathbf{x} \in U \cap \mathcal{A}(\mathbf{q})$. As $\mathbf{q}$ varies over $\Gamma$, we see that $\psi^{+} \leq z_{1}$ in $U$. Therefore the trace 
of $\psi^{+}$is strictly smaller than its prescribed Dirichlet boundary value (of $z_{2}$ ) on $\Gamma$ and so $T \psi^{+} \cdot \nu=1$ on $\Gamma$; this follows, for example, from [2,20]. Thus $\left(U, \psi^{+}\right)$is an upper Bernstein pair for $(\Gamma, H)$. Now define $\psi^{-}=-\psi^{+}$in $U$.

Remark 2.6. Suppose $\Omega$ is a $C^{2, \lambda}$ domain in $\mathbb{R}^{2}$ for some $\lambda \in(0,1), \mathbf{y} \in \partial \Omega$ and $\Lambda(\mathbf{y})<$ $2|H(\mathbf{y})|$, where $\Lambda(\mathbf{y})$ denotes the curvature of $\partial \Omega$ at $\mathbf{y}$. If $H$ is non-negative in $U \cap \Omega$ for some neighborhood $U$ of $\mathbf{y}$, then the argument which establishes [10, Corollary 14.13] and boundary regularity results (e.g., [2, 20]) imply that there exist $\delta>0$ and an upper Bernstein pair $\left(U^{+}, \psi^{+}\right)$for $(\Gamma, H)$, where $\Gamma=B_{\delta}(\mathbf{y}) \cap \partial \Omega$. If $H$ is non-positive in $U \cap \Omega$ for some neighborhood $U$ of $\mathbf{y}$, then there exist $\delta>0$ and a lower Bernstein pair $\left(U^{-}, \psi^{-}\right)$ for $(\Gamma, H)$, where $\Gamma=B_{\delta}(\mathbf{y}) \cap \partial \Omega$.

Proof of Theorem 1.1. We note, as in [16], that the conclusion of Theorem 1.1 is a local one and so, for small $\delta>0$, we can replace $\Omega$ by a $C^{2, \lambda}$ set $\Omega^{*}$ such that $\Omega \cap B_{\delta}(\mathbf{y})=\Omega^{*} \cap B_{\delta}(\mathbf{y})$ and $\Omega^{*} \subset B_{2 \delta}(\mathbf{y})$. We may assume $\Omega$ is a bounded domain. Set $S_{0}=\{(\mathbf{x}, f(\mathbf{x})): \mathbf{x} \in \Omega\}$. From the calculation on page 170 of [16], we see that the area of $S_{0}$ is finite; let $M_{0}$ denote this area. For $\delta \in(0,1)$, set

$$
p(\delta)=\sqrt{\frac{8 \pi M_{0}}{\ln (1 / \delta)}} .
$$

Let $E=\left\{(u, v): u^{2}+v^{2}<1\right\}$. As in [5, 16], there is a parametric description of the surface $S_{0}$

$$
Y(u, v)=(a(u, v), b(u, v), c(u, v)) \in C^{2}\left(E: \mathbb{R}^{3}\right)
$$

which has the following properties:

(a) $Y$ is a diffeomorphism of $E$ onto $S_{0}$.

$\left(\mathrm{a}_{2}\right)$ Set $G(u, v)=(a(u, v), b(u, v)),(u, v) \in E$. Then $G \in C^{0}\left(\bar{E}: \mathbb{R}^{2}\right)$.

(a) Set $\sigma(\mathbf{y})=G^{-1}(\partial \Omega \backslash\{\mathbf{y}\})$; then $\sigma(\mathbf{y})$ is a connected arc of $\partial E$ and $Y$ maps $\sigma(\mathbf{y})$ onto $\partial \Omega \backslash\{\mathbf{y}\}$. We may assume the endpoints of $\sigma(\mathbf{y})$ are $\mathbf{o}_{1}(\mathbf{y})$ and $\mathbf{o}_{2}(\mathbf{y})$. (Note that $\mathbf{o}_{1}(\mathbf{y})$ and $\mathbf{o}_{2}(\mathbf{y})$ are not assumed to be distinct.)

(a) $Y$ is conformal on $E: Y_{u} \cdot Y_{v}=0, Y_{u} \cdot Y_{u}=Y_{v} \cdot Y_{v}$ on $E$.

(as) $\triangle Y:=Y_{u u}+Y_{v v}=H(Y) Y_{u} \times Y_{v}$ on $E$.

From Lemma 2.4 when $H \equiv 0$ and Lemma 2.5 when $H$ satisfies 1.1 , we see that upper and lower Bernstein pairs $\left(U^{ \pm}, \psi^{ \pm}\right)$for $(\Gamma, H)$ exist. Notice that for each $C \in \mathbb{R}, Q\left(\psi^{+}+C\right)=$ $Q\left(\psi^{+}\right) \leq 0$ on $\Omega \cap U^{+}$and $Q\left(\psi^{-}+C\right)=Q\left(\psi^{-}\right) \geq 0$ on $\Omega \cap U^{-}$, and so

$$
N\left(\psi^{+}+C\right)(\mathbf{x}) \leq 2 H(\mathbf{x}, f(\mathbf{x}))=N f(\mathbf{x}) \quad \text { for } \mathbf{x} \in \Omega \cap U^{+}
$$


and

$$
N\left(\psi^{-}+C\right)(\mathbf{x}) \geq 2 H(\mathbf{x}, f(\mathbf{x}))=N f(\mathbf{x}) \quad \text { for } \mathbf{x} \in \Omega \cap U^{-} .
$$

Let $q$ denote a modulus of continuity for $\psi^{+}$and $\psi^{-}$.

Let $\zeta(\mathbf{y})=\partial E \backslash \sigma(\mathbf{y})$; then $G(\zeta(\mathbf{y}))=\{\mathbf{y}\}$ and $\mathbf{o}_{1}(\mathbf{y})$ and $\mathbf{o}_{2}(\mathbf{y})$ are the endpoints of $\zeta(\mathbf{y})$. There exists a $\delta_{1}>0$ such that if $\mathbf{w} \in E$ and $\operatorname{dist}(\mathbf{w}, \zeta(\mathbf{y})) \leq 2 \delta_{1}$, then $G(\mathbf{w}) \in$ $U^{+} \cap U^{-}$. Now $T \psi^{ \pm} \cdot \nu= \pm 1$ (in the sense of [3]) almost everywhere on an open subset $\Upsilon^{ \pm}$of $\partial U^{ \pm}$which contains $\bar{\Gamma}$; there exists a $\delta_{2}>0$ such that $\left(\partial U^{ \pm} \backslash \Upsilon^{ \pm}\right) \cap\left\{\mathbf{x} \in \mathbb{R}^{2}\right.$ : $\left.|\mathbf{x}-\mathbf{y}| \leq 2 p\left(\delta_{2}\right)\right\}=\emptyset$. Set $\delta^{*}=\min \left\{\delta_{1}, \delta_{2}\right\}$ and

$$
V^{*}=\left\{\mathbf{w} \in E: \operatorname{dist}(\mathbf{w}, \zeta(\mathbf{y}))<\delta^{*}\right\}
$$

Notice if $\mathbf{w} \in V^{*}$, then $G(\mathbf{w}) \in U^{+} \cap U^{-}$.

Claim. $Y$ is uniformly continuous on $V^{*}$ and so extends to a continuous function on $\overline{V^{*}}$.

Proof. Let $\epsilon>0$. Choose $\delta \in\left(0,\left(\delta^{*}\right)^{2}\right)$ such that $p(\delta)+2 q(p(\delta))<\epsilon$. Let $\mathbf{w}_{1}, \mathbf{w}_{2} \in V^{*}$ with $\left|\mathbf{w}_{1}-\mathbf{w}_{2}\right|<\delta$; then $G\left(\mathbf{w}_{1}\right), G\left(\mathbf{w}_{2}\right) \in U^{+} \cap U^{-}$. Set $C_{r}(\mathbf{w})=\{\mathbf{u} \in E:|\mathbf{u}-\mathbf{w}|=r\}$ and $B_{r}(\mathbf{w})=\{\mathbf{u} \in E:|\mathbf{u}-\mathbf{w}|<r\}$. From the Courant-Lebesgue Lemma (e.g., Lemma 3.1 in 4 ), we see that there exists $\rho=\rho(\delta) \in(\delta, \sqrt{\delta})$ such that the arc length $l_{\rho}\left(\mathbf{w}_{1}\right)$ of $Y\left(C_{\rho}\left(\mathbf{w}_{1}\right)\right)$ is less than $p(\delta)$. Notice that $\mathbf{w}_{2} \in B_{\rho(\delta)}\left(\mathbf{w}_{1}\right)$. Let

$$
k(\delta)\left(\mathbf{w}_{1}\right)=\inf _{\mathbf{u} \in C_{\rho(\delta)}\left(\mathbf{w}_{1}\right)} c(\mathbf{u})=\inf _{\mathbf{x} \in G\left(C_{\rho(\delta)}\left(\mathbf{w}_{1}\right)\right)} f(\mathbf{x})
$$

and

$$
m(\delta)\left(\mathbf{w}_{1}\right)=\sup _{\mathbf{u} \in C_{\rho(\delta)}\left(\mathbf{w}_{1}\right)} c(\mathbf{u})=\sup _{\mathbf{x} \in G\left(C_{\rho(\delta)}\left(\mathbf{w}_{1}\right)\right)} f(\mathbf{x})
$$

(recall that $c(\cdot)$ is the third component of $Y$ in $(2.3)$ ); then

$$
m(\delta)\left(\mathbf{w}_{1}\right)-k(\delta)\left(\mathbf{w}_{1}\right) \leq l_{\rho}<p(\delta) .
$$

Fix $\mathbf{x}_{0} \in C_{\rho(\delta)}^{\prime}\left(\mathbf{w}_{1}\right)$. Set

$$
C^{+}=\inf _{\mathbf{x} \in U^{+} \cap C_{\rho(\delta)}^{\prime}\left(\mathbf{w}_{1}\right)} \psi^{+}(\mathbf{x}) \text { and } C^{-}=\sup _{\mathbf{x} \in U^{-} \cap C_{\rho(\delta)}^{\prime}\left(\mathbf{w}_{1}\right)} \psi^{-}(\mathbf{x}) .
$$

Then $\psi^{+}-C^{+} \geq 0$ on $U^{+} \cap C_{\rho(\delta)}^{\prime}\left(\mathbf{w}_{1}\right)$ and $\psi^{-}-C^{-} \leq 0$ on $U^{-} \cap C_{\rho(\delta)}^{\prime}\left(\mathbf{w}_{1}\right)$. Therefore, for $\mathbf{x} \in U^{+} \cap U^{-} \cap C_{\rho(\delta)}^{\prime}\left(\mathbf{w}_{1}\right)$, we have

$$
k(\delta)\left(\mathbf{w}_{1}\right)+\left(\psi^{-}(\mathbf{x})-C^{-}\right) \leq f(\mathbf{x}) \leq m(\delta)\left(\mathbf{w}_{1}\right)+\left(\psi^{+}(\mathbf{x})-C^{+}\right) .
$$

Set

$$
b^{+}(\mathbf{x})=m(\delta)\left(\mathbf{w}_{1}\right)+\left(\psi^{+}(\mathbf{x})-C^{+}\right) \quad \text { for } \mathbf{x} \in U^{+} \cap \overline{G\left(B_{\rho(\delta)}\left(\mathbf{w}_{1}\right)\right)}
$$


and

$$
b^{-}(\mathbf{x})=k(\delta)\left(\mathbf{w}_{1}\right)+\left(\psi^{-}(\mathbf{x})-C^{-}\right) \quad \text { for } \mathbf{x} \in U^{-} \cap \overline{G\left(B_{\rho(\delta)}\left(\mathbf{w}_{1}\right)\right)} .
$$

Now $\rho(\delta)<\sqrt{\delta}<\delta^{*} \leq \delta_{2}$; notice that if $\mathbf{w} \in \overline{B_{\rho(\delta)}\left(\mathbf{w}_{1}\right)}$, then $\left|\mathbf{w}-\mathbf{w}_{1}\right|<\delta_{2}$ and $|G(\mathbf{w})-\mathbf{y}|<2 p\left(\delta_{2}\right)$ and thus if $\mathbf{x} \in \overline{G\left(B_{\rho(\delta)}\left(\mathbf{w}_{1}\right)\right)} \cap \partial U^{ \pm}$, then $\mathbf{x} \in \Upsilon^{ \pm}$. From (2.4) and 2.5), the facts that $b^{-} \leq f$ on $U^{-} \cap C_{\rho(\delta)}^{\prime}\left(\mathbf{w}_{1}\right)$ and $f \leq b^{+}$on $U^{+} \cap C_{\rho(\delta)}^{\prime}\left(\mathbf{w}_{1}\right)$ and the general comparison principle (see [8, Theorem 5.1]), we have

$$
b^{-} \leq f \quad \text { on } U^{-} \cap \overline{G\left(B_{\rho(\delta)}\left(\mathbf{w}_{1}\right)\right)}
$$

and

$$
f \leq b^{+} \quad \text { on } U^{+} \cap \overline{G\left(B_{\rho(\delta)}\left(\mathbf{w}_{1}\right)\right)} .
$$

Since the diameter of $G\left(B_{\rho(\delta)}\left(\mathbf{w}_{1}\right)\right) \leq p(\delta)$, we have $\left|\psi^{ \pm}(\mathbf{x})-C^{ \pm}\right| \leq q(p(\delta))$ for $\mathbf{x} \in U^{ \pm} \cap G\left(B_{\rho(\delta)}\left(\mathbf{w}_{1}\right)\right)$; recall that $q(\cdot)$ is a modulus of continuity for $\psi^{+}$and $\psi^{-}$. Thus, whenever $\mathbf{x}_{1}, \mathbf{x}_{2} \in \overline{G\left(B_{\rho(\delta)}\left(\mathbf{w}_{1}\right)\right)}$, we have $\mathbf{x}_{1}, \mathbf{x}_{2} \in U^{+} \cap U^{-}$. Since $c(\mathbf{w})=f(G(\mathbf{w}))$, $G\left(\mathbf{w}_{1}\right) \in U^{+} \cap U^{-}$and $G\left(\mathbf{w}_{2}\right) \in U^{+} \cap U^{-}$, we have

$$
b^{-}\left(G\left(\mathbf{w}_{1}\right)\right)-b^{+}\left(G\left(\mathbf{w}_{2}\right)\right) \leq c\left(\mathbf{w}_{1}\right)-c\left(\mathbf{w}_{2}\right) \leq b^{+}\left(G\left(\mathbf{w}_{1}\right)\right)-b^{-}\left(G\left(\mathbf{w}_{2}\right)\right)
$$

or

$$
\begin{aligned}
& -\left[m(\delta)\left(\mathbf{w}_{1}\right)-k(\delta)\left(\mathbf{w}_{1}\right)+\left(\psi^{+}\left(G\left(\mathbf{w}_{2}\right)\right)-C^{+}\right)-\left(\psi^{-}\left(G\left(\mathbf{w}_{1}\right)\right)+C^{-}\right)\right] \\
\leq & c\left(\mathbf{w}_{1}\right)-c\left(\mathbf{w}_{2}\right) \\
\leq & {\left[m(\delta)\left(\mathbf{w}_{1}\right)-k(\delta)\left(\mathbf{w}_{1}\right)+\left(\psi^{+}\left(G\left(\mathbf{w}_{1}\right)\right)-C^{+}\right)-\left(\psi^{-}\left(G\left(\mathbf{w}_{2}\right)\right)+C^{-}\right)\right] . }
\end{aligned}
$$

Since $\left|\psi^{ \pm}(G(\mathbf{w}))-C^{ \pm}\right| \leq q(p(\delta))$ for $\mathbf{w} \in B_{\rho(\delta)}\left(\mathbf{w}_{1}\right) \cap U^{ \pm}$, we have

$$
\left|c\left(\mathbf{w}_{1}\right)-c\left(\mathbf{w}_{2}\right)\right| \leq p(\delta)+2 q(p(\delta))<\epsilon .
$$

Thus $c$ is uniformly continuous on $V^{*}$ and, since $G \in C^{0}\left(\bar{E}: \mathbb{R}^{2}\right)$, we see that $Y$ is uniformly continuous on $V^{*}$. Therefore $Y$ extends to a continuous function, still denote $Y$, on $\overline{V^{*}}$.

Notice that

$$
\lim _{\Gamma_{1} \ni \mathbf{x} \rightarrow \mathbf{y}} f(\mathbf{x})=\lim _{\partial E \ni \mathbf{w} \rightarrow \mathbf{o}_{1}(\mathbf{y})} c(\mathbf{w})=c\left(\mathbf{o}_{1}(\mathbf{y})\right)
$$

and

$$
\lim _{\Gamma_{2} \ni \mathbf{x} \rightarrow \mathbf{y}} f(\mathbf{x})=\lim _{\partial E \ni \mathbf{w} \rightarrow \mathbf{o}_{2}(\mathbf{y})} c(\mathbf{w})=c\left(\mathbf{o}_{2}(\mathbf{y})\right)
$$

and so, with $z_{1}=c\left(\mathbf{o}_{1}(\mathbf{y})\right)$ and $z_{2}=c\left(\mathbf{o}_{2}(\mathbf{y})\right)$, we see that the limits

$$
\lim _{\Gamma_{1} \ni \mathbf{x} \rightarrow \mathbf{y}} f(\mathbf{x})=z_{1} \quad \text { and } \quad \lim _{\Gamma_{2} \ni \mathbf{x} \rightarrow \mathbf{y}} f(\mathbf{x})=z_{2}
$$

exist.

Now we need to consider two cases: 
(A) $\mathbf{o}_{1}(\mathbf{y})=\mathbf{o}_{2}(\mathbf{y})$.

(B) $\mathbf{o}_{1}(\mathbf{y}) \neq \mathbf{o}_{2}(\mathbf{y})$.

These correspond to Cases 5 and 3 respectively in Step 1 of the proof of [16, Theorem 1]. Case (A). Suppose $\mathbf{o}_{1}(\mathbf{y})=\mathbf{o}_{2}(\mathbf{y})$; set $\mathbf{o}=\mathbf{o}_{1}(\mathbf{y})=\mathbf{o}_{2}(\mathbf{y})$. Then $f$ extends to a function in $C^{0}(\Omega \cup\{\mathbf{y}\})$ and Theorem 1.1(i) holds.

Proof. Notice that $G$ is a bijection of $E \cup\{\mathbf{o}\}$ and $\Omega \cup\{\mathbf{y}\}$. Thus we may define $f=c \circ G^{-1}$, so $f(G(\mathbf{w}))=c(\mathbf{w})$ for $\mathbf{w} \in E \cup\{\mathbf{o}\}$; this extends $f$ to a function defined on $\Omega \cup\{\mathbf{y}\}$. Let $\left\{\delta_{i}\right\}$ be a decreasing sequence of positive numbers converging to zero and consider the sequence of open sets $\left\{G\left(B_{\rho(i)}(\mathbf{o})\right)\right\}$ in $\Omega$, where $\rho(i)=\rho\left(\delta_{i}(\mathbf{o})\right)$. Now $\mathbf{y} \notin G\left(C_{\rho(i)}(\mathbf{o})\right)$ and so there exist $\sigma_{i}>0$ such that

$$
P(i)=\left\{\mathbf{x} \in \Omega:|\mathbf{x}-\mathbf{y}|<\sigma_{i}\right\} \subset G\left(B_{\rho(i)}(\mathbf{o})\right)
$$

for each $i \in \mathbb{N}$. Thus if $\mathbf{x} \in P(i)$, we have $|f(\mathbf{x})-f(\mathbf{y})|<p\left(\delta_{i}\right)+2 q\left(p\left(\delta_{i}\right)\right)$. The continuity of $f$ at $\mathbf{y}$ follows from this.

Case (B). Suppose $\mathbf{o}_{1}(\mathbf{y}) \neq \mathbf{o}_{2}(\mathbf{y})$. Then Theorem 1.1(ii) holds.

Proof. As at the end of Step 1 of the proof of $\left[16\right.$, Theorem 1], we define $X: B \rightarrow \mathbb{R}^{3}$ by $X=Y \circ g$ and $K: B \rightarrow \mathbb{R}^{2}$ by $K=G \circ g$, where $B=\left\{(u, v) \in \mathbb{R}^{2}: u^{2}+v^{2}<1, v>0\right\}$ and $g: \bar{B} \rightarrow \bar{E}$ is either a conformal or an indirectly conformal (or anticonformal) map from $\bar{B}$ onto $\bar{E}$ such that $g(1,0)=\mathbf{o}_{1}(\mathbf{y}), g(-1,0)=\mathbf{o}_{2}(\mathbf{y})$ and $g(u, 0) \in \mathbf{o}_{1}(\mathbf{y}) \mathbf{o}_{2}(\mathbf{y})$ for each $u \in[-1,1]$, where $\mathbf{a b}$ denotes the (appropriate) choice of arc in $\partial E$ with $\mathbf{a}$ and $\mathbf{b}$ as endpoints.

Notice that $K(u, 0)=\mathbf{y}$ for $u \in[-1,1]$. Set $x=a \circ g, y=b \circ g$ and $z=c \circ g$, so that $X(u, v)=(x(u, v), y(u, v), z(u, v))$ for $(u, v) \in B$. Now, from Step 2 of the proof of [16, Theorem 1],

$$
X \in C^{0}\left(\bar{B}: \mathbb{R}^{3}\right) \cap C^{1, \iota}\left(B \cup\{(u, 0):-1<u<1\}: \mathbb{R}^{3}\right)
$$

for some $\iota \in(0,1)$ and $X(u, 0)=(\mathbf{y}, z(u, 0))$ cannot be constant on any nondegenerate interval in $[-1,1]$. Define $\Theta(u)=\arg \left(x_{v}(u, 0)+i y_{v}(u, 0)\right)$. From equation 16, (12)], we see that

$$
\alpha_{1}=\lim _{u \downarrow-1} \Theta(u) \quad \text { and } \quad \alpha_{2}=\lim _{u \uparrow 1} \Theta(u) ;
$$

here $\alpha_{1}<\alpha_{2}$. As in Steps 2-5 of the proof of [16, Theorem 1], we see that $R f(\theta)$ exists when $\theta \in\left(\alpha_{1}, \alpha_{2}\right)$,

$$
\begin{array}{ccc}
\overline{G^{-1}\left(L\left(\alpha_{2}\right)\right)} \cap \partial E=\left\{\mathbf{o}_{1}(\mathbf{y})\right\} \quad\left(\text { and } \overline{K^{-1}\left(L\left(\alpha_{2}\right)\right)} \cap \partial B=\{(1,0)\}\right) & \text { when } \alpha_{2}<\beta(\mathbf{y}), \\
\overline{G^{-1}\left(L\left(\alpha_{1}\right)\right)} \cap \partial E=\left\{\mathbf{o}_{2}(\mathbf{y})\right\} \quad\left(\text { and } \overline{K^{-1}\left(L\left(\alpha_{1}\right)\right)} \cap \partial B=\{(-1,0)\}\right) & \text { when } \alpha_{1}>\alpha(\mathbf{y}),
\end{array}
$$


where $L(\theta)=\left\{\mathbf{y}+(r \cos (\theta), r \sin (\theta)) \in \Omega: 0<r<\delta^{*}\right\}$, and we see that $R f$ is strictly increasing or strictly decreasing on $\left(\alpha_{1}, \alpha_{2}\right)$. We may argue as in Case $\mathrm{A}$ to see that $f$ is uniformly continuous on

$$
\Omega^{+}=\left\{\mathbf{y}+(r \cos (\theta), r \sin (\theta)) \in \Omega: 0<r<\delta, \alpha_{2} \leq \theta<\beta(\mathbf{y})+\epsilon\right\}
$$

and $f$ is uniformly continuous on

$$
\Omega^{-}=\left\{\mathbf{y}+(r \cos (\theta), r \sin (\theta)) \in \Omega: 0<r<\delta, \alpha(\mathbf{y})-\epsilon<\theta \leq \alpha_{1}\right\}
$$

for some small $\epsilon>0$ and $\delta>0$, since $G$ is a bijection of $E \cup\left\{\mathbf{o}_{1}(\mathbf{y})\right\}$ and $\Omega \cup\{\mathbf{y}\}$ and a bijection of $E \cup\left\{\mathbf{o}_{2}(\mathbf{y})\right\}$ and $\Omega \cup\{\mathbf{y}\}$. Theorem 1.1 then follows, as in [7, from Steps 2-5 of the proof of [16, Theorem 1].

Proof of Corollary 1.3. From Theorem 1.1, we see that the radial limits $R f(\theta, \mathbf{y})$ exist for each $\theta \in[\alpha(\mathbf{y}), \beta(\mathbf{y})]$. Set $z_{1}=R f(\alpha(\mathbf{y}), \mathbf{y}), z_{2}=R f(\beta(\mathbf{y}), \mathbf{y})$ and $z_{3}=\phi(\mathbf{y})$. If $z_{1}=z_{2}$, then Theorem 1.1(i) holds. (If $f$ is symmetric with respect to a line through $\mathbf{y}$, then $z_{1}=z_{2}$ and we are done.)

Suppose otherwise that $z_{1} \neq z_{2}$; we may assume that $z_{1}<z_{3}$ and $z_{1}<z_{2}$. Then there exist $\alpha_{1}, \alpha_{2} \in[\alpha(\mathbf{y}), \beta(\mathbf{y})]$ with $\alpha_{1}<\alpha_{2}$ such that

$$
R f(\theta, \mathbf{y}) \text { is } \begin{cases}\text { constant }\left(=z_{1}\right) & \text { for } \alpha(\mathbf{y}) \leq \theta \leq \alpha_{1}, \\ \text { strictly increasing } & \text { for } \alpha_{1} \leq \theta \leq \alpha_{2}, \\ \text { constant }\left(=z_{2}\right) & \text { for } \alpha_{2} \leq \theta \leq \beta(\mathbf{y}) .\end{cases}
$$

From Theorem 1.1, we see that $R f(\theta, \mathbf{y})$ exists for each $y \in \Gamma$ and $\theta \in[\alpha(\mathbf{y}), \beta(\mathbf{y})]$ and $f$ is continuous on $\Omega \cup \Gamma \backslash \Upsilon$ for some countable subset $\Upsilon$ of $\Gamma$. Let $z_{0} \in\left(z_{1}, \min \left\{z_{2}, z_{3}\right\}\right)$ and $\theta_{0} \in\left(\alpha_{1}, \alpha_{2}\right)$ satisfy $R f\left(\theta_{0}, \mathbf{y}\right)=z_{0}$. Let $C_{0} \subset \Omega$ be the $z_{0}$-level curve of $f$ which has $\mathbf{y}$ and a point $\mathbf{y}_{0} \in \partial \Omega \backslash\{\mathbf{y}\}$ as endpoints. Let $\mathbf{y}_{1} \in \Gamma_{1}(\mathbf{y}) \cap \Gamma \backslash \Upsilon$ and $\mathbf{y}_{2} \in C_{0}$ such that the (open) line segment $L$ joining $\mathbf{y}_{1}$ and $\mathbf{y}_{2}$ is entirely contained in $\Omega$; recall that $\Gamma_{1}(\mathbf{y})$ is the "lower" component of $\partial \Omega \cap B_{\delta}(\mathbf{y}) \backslash\{\mathbf{y}\}$ (see Figure 1.1). Let $M<\min \left\{z_{1}, \inf _{L} f\right\}$. Let $\Pi$ be the plane containing $\left(\mathbf{y}, z_{0}\right)$ and $L \times\{M\}$ and let $h$ be the affine function on $\mathbb{R}^{2}$ whose graph is $\Pi$. Let $\Omega_{0}$ be the component of $\Omega \backslash\left(C_{0} \cup L\right)$ whose closure contains $B_{\delta}(\mathbf{y}) \cap \Gamma_{1}(\mathbf{y})$ for small $\delta>0$. By first choosing $\mathbf{y}_{2}$ sufficiently near $\mathbf{y}$ and then choosing $\mathbf{y}_{1}$ sufficiently near $\mathbf{y}$, we may assume that $\mathbf{y}$ is the furthest point on $C_{0}$ between $\mathbf{y}$ and $\mathbf{y}_{2}$ away from $L$. Then $h=M<f$ on $L, h \leq z_{0}=f$ on the portion of $C_{0}$ between $\mathbf{y}$ and $\mathbf{y}_{2}$, and $h(\mathbf{y})=z_{0}>R f(\theta, \mathbf{y})$ for each $\theta \in\left[\alpha(\mathbf{y}), \theta_{0}\right)$. Thus $h \leq f$ on $\Omega \cap \partial \Omega_{0}$ and $h>f$ on $B_{\delta}(\mathbf{y}) \cap \partial \Omega_{0}$ for some sufficiently small $\delta>0$.

Then there is a curve $C \subset \Omega_{0}$ on which $f=h$ whose endpoints are $\mathbf{y}_{3}$ and $\mathbf{y}$, for some $\mathbf{y}_{3} \in \Gamma_{1}(\mathbf{y})$ between $\mathbf{y}_{1}$ and $\mathbf{y}$, such that $h>f$ in $\Omega_{1}$, where $\Omega_{1} \subset \Omega_{0}$ is the open set 
bounded by $C$ and the portion of $\Gamma_{1}(\mathbf{y})$ between $\mathbf{y}$ and $\mathbf{y}_{3}$. (In Figure 2.2, on the left, $\{(\mathbf{x}, h(\mathbf{x})): \mathbf{x} \in C\}$ is in red, $L$ is in dark blue, $C_{0}$ is in yellow, and the light blue region is a portion of $\partial_{\mathbf{y}}^{1} \Omega \times \mathbb{R}$, and, on the right, $\Omega_{1}$ is in light green and $\Gamma_{2}(\mathbf{y})$ is in magenta.)
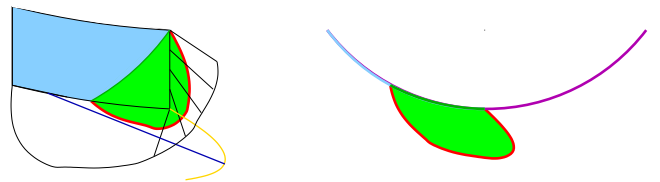

Figure 2.2: Side View of $\Pi \cap(\Omega \times \mathbb{R})$ (left) and $\Omega_{1}$ (right).

Now let $g \in C^{2}(\Omega)$ be defined by $g=f$ on $\Omega \backslash \overline{\Omega_{1}}$ and $g=h$ on $\Omega_{1}$ and observe that $J(g)<J(f)$. (The functional $J(f)$ includes the area of the blue surface in Figure 2.3 . which is a subset of $\partial \Omega \times \mathbb{R}$, and the area of the purple surface of this figure, which is the subset of the graph of $f$ over $\Omega_{1}$ while $J(g)$ does not include the areas of the blue and purple surfaces and instead includes the area of the green surface on the left side of Figure 2.2, which is the portion of the plane $\Pi$ over $\Omega_{1}$.)

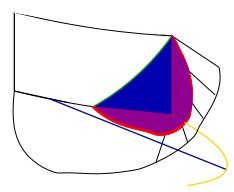

Figure 2.3: Graph of $f$ over $\Omega_{1}$ (purple) and part of $\partial \Omega \times \mathbb{R}$ (blue).

This contradicts the fact that $f$ minimizes $J$. Thus it must be the case that $z_{1}=z_{2}$, Theorem 1.1 (i) holds and $f$ is continuous at $\mathbf{y}$. (Notice that the set $\Omega_{1}=\left\{\mathbf{x} \in \Omega_{0}: h(\mathbf{x})>\right.$ $f(\mathbf{x})\}$ could be more complex but the proof is unchanged; if $V$ is an open set in $\Omega_{0}$ with $h<f$ in $V, h=f$ on $\partial V \cap \Omega$ and $h \leq f$ on $\partial V \cap \partial \Omega$ is an "inclusion" in $\Omega_{1}$, it does not matter; we still set $g=h$ on $\Omega_{1}$ and $g=f$ on $\Omega \backslash \Omega_{1}$.)

Proof of Corollary 1.2. The proof of Corollary 1.2 is essentially the same as that of Corollary 1.3 we replace $f$ with a function $g$ and obtain a contradiction by showing $J(g)<J(f)$, where

$$
J(u)=\int_{\Omega} \sqrt{1+|D u|^{2}} d \mathbf{x}+\int_{\Omega}\left(\int_{c}^{u(\mathbf{x})} 2 H(\mathbf{x}, t) d t\right) d \mathbf{x}+\int_{\partial \Omega}|u-\phi| d s
$$

for $u \in B V(\Omega)$.

As in the proof of Corollary 1.3, we assume $z_{1}<z_{3}, z_{1}<z_{2}$, and $R f(\theta, \mathbf{y})$ is strictly increasing on $\left(\alpha_{1}, \alpha_{2}\right)$ and constant on $\left[\alpha(\mathbf{y}), \alpha_{1}\right]$ and $\left[\alpha_{2}, \beta(\mathbf{y})\right]$. Let $z_{0} \in\left(z_{1}, \min \left\{z_{2}, z_{3}\right\}\right)$ and $\theta_{0} \in\left(\alpha_{1}, \alpha_{2}\right)$ satisfy $R f\left(\theta_{0}, \mathbf{y}\right)=z_{0}$. Extend $H$ so $H \in C^{1}\left(\mathbb{R}^{2}\right)$. Let $R>0$ be 
small enough that $2 R|H(\mathbf{x})| \leq 1$ for all $\mathbf{x} \in B_{2 R}(\mathbf{y})$. Set $\theta_{b}=\left(\theta_{0}+\beta(\mathbf{y})\right) / 2$ and $T=$ $\left\{\mathbf{y}+r\left(\cos \theta_{b}, \sin \theta_{b}\right): r \in \mathbb{R}\right\}$, and let $C(R)$ be the circle of radius $R$ which passes through $\mathbf{y}$, is tangent at $\mathbf{y}$ to the line $T$, and intersects $\Gamma_{1}(\mathbf{y})$. Let $V(R)$ be the open disk inside $C(R)$. (In Figure 2.4, the blue arcs are part of $\partial \Omega$, locally $\Omega$ lies below these blue arcs, the red rays represent $\theta=\theta_{0}$, and, on the right, $V(R)$ is the yellow disk, $C(R)$ is the black circle, and $T$ is the green line.) Notice that $(1.2)-(1.3)$ in the domain $V(R)$ is solvable for all $\phi \in C^{0}(C(R))$.
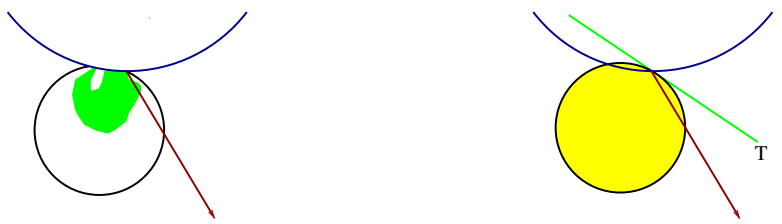

Figure 2.4: $U$ (green region; left); $C(R)$ (black circle; right).

Let $h \in C^{2}(\overline{V(R)})$ satisfy $Q h=0$ in $V(R), h(\mathbf{y})=z_{0}, h<f$ on $C(R) \cap \Omega$ (recall $\left.R f\left(\theta_{b}, \mathbf{y}\right)>R f\left(\theta_{0}, \mathbf{y}\right)=z_{0}\right)$, and $h \leq z_{0}$ on $C(R) \backslash \Omega$. Set $U=\{\mathbf{x} \in \Omega \cap V(R): h(\mathbf{x})>$ $f(\mathbf{x})\}$ (see Figure 2.4 and notice that $\Gamma_{1}(\mathbf{y}) \cap B_{\delta}(\mathbf{y}) \subset \bar{U} \subset V(R) \cup\{\mathbf{y}\}$ for some $\delta>0$. Now define $g \in C^{0}(\Omega)$ by $g=h$ on $U$ and $g=f$ on $\Omega \backslash U$. Then $J(g)<J(f)$ as before in the proof of Corollary 1.3 .

Proof of Example 1.4. By Corollary 1.2, $f$ is continuous on $\Omega \cup\{(0,0)\}$. Clearly $f$ is continuous at $(x, y)$ when $(x+1)^{2}+y^{2}=\cosh ^{2}(1)$. By [24], $f$ is continuous at $(x, y)$ when $(x+1)^{2}+y^{2}=1$ and $(x, y) \neq(0,0)$. The parametrization 2.3) of the graph of $f$ (restricted to $\Omega \backslash\{(x, 0): x<0\}$ ) satisfies $Y \in C^{0}(\bar{E})$. Notice that $\zeta((0,0))=\{\mathbf{o}\}$ (since $\beta((0,0))-\alpha((0,0))=\pi$ and $\left.z_{1}=z_{2}\right)$ for some $\mathbf{o} \in \partial E$. Suppose $G$ in $\left(\mathrm{a}_{2}\right)$ is not one-to-one. Then there exists a nondegenerate arc $\zeta \subset \partial E$ such that $G(\zeta)=\left\{\mathbf{y}_{1}\right\}$ for some $\mathbf{y}_{1} \in \partial \Omega$ and therefore $f$ is not continuous at $\mathbf{y}_{1}$, which is a contradiction. Thus $f=g \circ G^{-1}$ and so $f \in C^{0}(\bar{\Omega})$. (The continuity of $G^{-1}$ follows, for example, from Lemma 3.1 in $[4$.)

Remark 2.7. The term "order of non-uniformity", used in [17], and the term genre, established in 1912 by Bernstein, adopted by Serrin (see [23, p. 425]) and used in [13], are numbers which measure the variation from uniform ellipticity of a quasilinear elliptic operator; the genre and the "order of non-uniformity" for the minimal surface operator are both equal to two (2) while the genre and the "order of non-uniformity" for uniformly elliptic operators are both equal to zero (0). We are confused by the invention for a new phrase for an existing and named phenomenon. 


\section{Acknowledgments}

The second author wishes to thank the Institute of Mathematics at Academia Sinica for support during part of this investigation and to thank Professor Fei-tsen Liang for helpful discussions.

\section{References}

[1] M. Bergner, On the Dirichlet problem for the prescribed mean curvature equation over general domains, Differential Geom. Appl. 27 (2009), no. 3, 335-343.

[2] T. Bourni, $C^{1, \alpha}$ theory for the prescribed mean curvature equation with Dirichlet data, J. Geom. Anal. 21 (2011), no. 4, 982-1035.

[3] P. Concus and R. Finn, On capillary free surfaces in the absence of gravity, Acta Math. 132 (1974), 177-198.

[4] R. Courant, Dirichlet's Principle, Conformal Mapping, and Minimal Surfaces, Interscience, New York, 1950.

[5] A. R. Elcrat and K. E. Lancaster, Boundary behavior of a nonparametric surface of prescribed mean curvature near a reentrant corner, Trans. Amer. Math. Soc. 297 (1986), no. 2, 645-650.

[6] _ Bernstein functions and the Dirichlet problem, SIAM J. Math. Anal. 20 (1989), no. 5, 1055-1068.

[7] M. Entekhabi and K. Lancaster, Radial limits of bounded nonparametric prescribed mean curvature surfaces, Pacific J. Math. 283 (2016), no. 2, 341-351.

[8] R. Finn, Equilibrium Capillary Surfaces, Grundlehren der Mathematischen Wissenschaften 284, Springer-Verlag, New York, 1986.

[9] _ Moon surfaces, and boundary behaviour of capillary surfaces for perfect wetting and non-wetting, Proc. Lond. Math. Soc. s3-57 (1988), no. 3, 542-576.

[10] D. Gilbarg and N. Trudinger, Elliptic Partial Differential Equations of Second Order, (revised second edition), Grundlehren der Mathematischen Wissenschaften 224, Springer-Verlag, New York, 1998.

[11] K. Hayasida and M. Nakatani, On the Dirichlet problem of prescribed mean curvature equations without H-convexity condition, Nagoya Math. J 157 (2000), 177-209. 
[12] H. Jenkins and J. Serrin, The Dirichlet problem for the minimal surface equation in higher dimensions, J. Reine Angew. Math. 229 (1968), 170-187.

[13] Z. Jin and K. Lancaster, Theorems of Phragmén-Lindelöf type for quasilinear elliptic equations, J. Reine Angew. Math. 514 (1999), 165-197.

[14] A. Korn, Über Minimalflächen, deren Randkurven wenig von ebenen Kurven abweichen [On minimal surfaces whose boundary curves deviate little from plane curves], Abh. Königl. Preuss. Akad. Wiss., Berlin, Phys.-Math. Cl. II (1909), 1-37.

[15] K. E. Lancaster, Existence and nonexistence of radial limits of minimal surfaces, Proc. Amer. Math. Soc. 106 (1989), no. 3, 757-762.

[16] K. E. Lancaster and D. Siegel, Existence and behavior of the radial limits of a bounded capillary surface at a corner, Pacific J. Math. 176 (1996), no. 1, 165-194. Correction to: "Existence and behavior of the radial limits of a bounded capillary surface at a corner", Pacific J. Math. 179 (1997), no. 2 , 397-402.

[17] C. P. Lau, Quasilinear elliptic equations with small boundary data, Manuscripta Math. 53 (1985), no. 1-2, 77-99.

[18] F.-T. Liang, An absolute gradient bound for nonparametric surfaces of constant mean curvature, Indiana Univ. Math. J. 41 (1992), no. 3, 569-604.

[19] _ Moon hypersurfaces and some related existence results of capillary hypersurfaces without gravity and of rotational symmetry, Pacific J. Math. 172 (1996), no. 2, 433-460.

[20] F.-H. Lin, Behaviour of nonparametric solutions and free boundary regularity, in: Miniconference on Geometry and Partial Differential Equations 2 (Canberra, 1986), 96-116, Proc. Centre Math. Aanl. Austral. Nat. Univ. 12, Austral. Nat. Univ., Canberra, 1987.

[21] J. C. C. Nitsche, Lectures on Minimal Surfaces I, Cambridge University Press, Cambridge, 1989.

[22] J. Ripoll and F. Tomi, On solutions to the exterior Dirichlet problem for the minimal surface equation with catenoidal ends, Adv. Calc. Var. 7 (2014), no. 2, 205-226.

[23] J. Serrin, The problem of Dirichlet for quasilinear elliptic differential equations with many independent variables, Philos. Trans. Roy. Soc. London Ser. A 264 (1969), $413-496$. 
[24] L. Simon, Boundary regularity for solutions of the non-parametric least area problem, Ann. of Math. (2) 103 (1976), no. 3, 429-455.

[25] G. H. Williams, The Dirichlet problem for the minimal surface equation with Lipschitz continuous boundary data, J. Reine Angew. Math. 354 (1984), 123-140.

[26] - The Dirichlet problem for the minimal surface equation, in: Miniconference on Nonlinear Analysis (Canberra, 1984), 233-239, Proc. Centre Math. Anal. Austral. Nat. Univ. 8, Austral. Nat. Univ., Canberra, 1984.

Mozhgan Nora Entekhabi

Department of Mathematics, Florida A \& M University, Tallahassee, FL 32307, USA

E-mail address: mozhgan.entekhabi@famu.edu

Kirk E. Lancaster

Wichita, Kansas 67226, USA

E-mail address: redwoodsrunner@gmail.com 